\title{
LA DIMENSIÓN DE GÉNERO EN EL DESARROLLO
}

Arizaldo Carvajal Burbano 


\title{
RESUMEN
}

\begin{abstract}
El artículo aborda el desarrollo desde la perspectiva de género. Presenta cuatro dimensiones básicas: una conceptualización sobre género y su relación con el desarrollo; el papel de la mujer en el desarrollo, las necesidades prácticas y estratégicas, y el empoderamiento de la mujer como actor del desarrollo. Trabajar esta perspectiva implica un mayor posicionamiento ético y político.
\end{abstract}

\section{PRESENTACIÓN}

"No hay desarrollo humano sin las mujeres y que la democracia implica, en primer término, la democracia genérica".

Marcela Lagarde

"Ten cuidado con el presente que construyes, debe parecerse al futuro que sueñas"

Mujeres creando, Bolivia

D odríamos atrevernos a decir que en Colombia "la inclusión del género en general y de las mujeres en particular en el estudio del desarrollo ha comenzado de forma relativamente lenta". Este paulatino interés por la mujer y el desarrollo aún no ha dado todos sus frutos. El reconocimiento explícito del importante papel que cumple la mujer en los procesos de desarrollo, según Santos y Caballero (1994:346), se debe básicamente a tres factores: la divulgación de la situación y posición de la mujer en la sociedad, la reformulación de las políticas de desarrollo y el reconocimiento internacional de la necesidad de incluir la perspectiva de género en los programas de cooperación al desarrollo.

Así, este escrito ${ }^{1}$ tiene como objetivo presentar algunos puntos de vista acerca del desarrollo desde la perspectiva de género. Se presentan cuatro aspectos: en primer lugar,

${ }^{1}$ Este artículo se realizó con base en el Documento "Género y desarrollo" (Inédito), elaborado en Madrid en 2001, cuando cursaba el Doctorado en Antropología Social en la Universidad Complutense de Madrid, con una Comisión de Estudios de Univalle. 
una discusión sobre la dimensión de género en el desarrollo. Un aspecto más concreto, como es el de mujer y desarrollo, se analiza en la segunda parte; la tercera muestra las necesidades del género en el desarrollo; y un tema significativo y polémico como es el empoderamiento, se trata en la cuarta. Este trabajo -que muestra aspectos globales de la problemática; puntos para la discusión-, pretende ser un aporte a un campo (teóricopráctico) que cada día está adquiriendo más importancia y significado en nuestra sociedad.

\section{LA DIMENSIÓN DE GÉNERO EN EL DESARROLLO}

El concepto de género -como el de desarrollo- es polémico y polisémico. Expresa Gabriela Castellanos que durante muchos años "la teoría feminista ha insistido en que las identidades femeninas y masculinas, así como las relaciones sociales entre hombres y mujeres, responden fundamentalmente a condicionamientos culturales, y no a la tiranía de una supuesta naturaleza biológica, invariable, ahistórica" (Castellanos, 2002:17). Así, el concepto de género es una contestación que opone cultura versus naturaleza, y "la idea de género surge en el pensamiento feminista como una reacción frente a la constatación de que las discriminaciones, la inferiorización y la subordinación de las mujeres se construye, justifica y legitima sobre la base de determinadas diferencias consideradas naturales e inmutables" (López y Sierra: 2001:1). El género es parte del contexto sociocultural. Es importante recordar que los estudios de género van más allá de una división de sexos (género no es igual a mujer) y que es fundamental analizar las relaciones sociales que se establecen entre hombres y mujeres.

Es especialmente importante tener en cuenta que la dimensión de género no ha de entenderse en la forma tradicional, como la línea biológica divisoria entre sexos, sino más bien en términos de las condiciones sociales y económicas que caracterizan las relaciones entre hombres y mujeres (NORAD, 1997:62).

Como vemos, la categoría de género, impulsada por el pensamiento feminista, fue creada para explicar que los roles sociales asignados y ejercidos por las mujeres y los hombres no son producto de diferencias biológicas "naturales" ni de sexo, sino el resultado de construcciones sociales y culturales asumidas históricamente.

Desde esta consideración, se entiende que la subordinación a la cual ha estado sometida la mitad de la población mundial -las mujeres- a través de diversos períodos históricos es producto de específicas formas de organización de las sociedades donde lo femenino y lo masculino no es el resultado de una definición biológica sino la consecuencia de una desigual e injusta jerarquización al respecto de las prácticas sociales, las funciones y la ubicación que tengamos en la sociedad (PNUD).

En otras palabras, según De Barbieri (citado en PNUD), los sistemas de género/sexo son los conjuntos de prácticas, símbolos, representaciones, normas y valores sociales 
que las sociedades elaboran a partir de la diferencia sexual-anátomo fisiológica y que dan sentido a la satisfacción de los impulsos sexuales, a la reproducción de la especie humana $y$, en general, al relacionamiento entre las personas.

La categoría de género además ha permitido demostrar que la desigualdad entre los sexos es una condición modificable. El análisis de las relaciones entre los géneros es el análisis de las relaciones de poder entre los hombres y las mujeres donde el género que posee el poder lo emplea para determinar el comportamiento del que no lo posee (PNUD).

El género es dinámico, es cambiante; el género es específico de cada cultura. En este sentido comporta un fuerte componente político e ideológico, transformador. Veamos la siguiente gráfica:

GRÁFICA No. 1

\section{LA PERSPECTIVA TRANSFORMADORA DE GÉNERO: ESQUEMA DE CONTENIDOS}

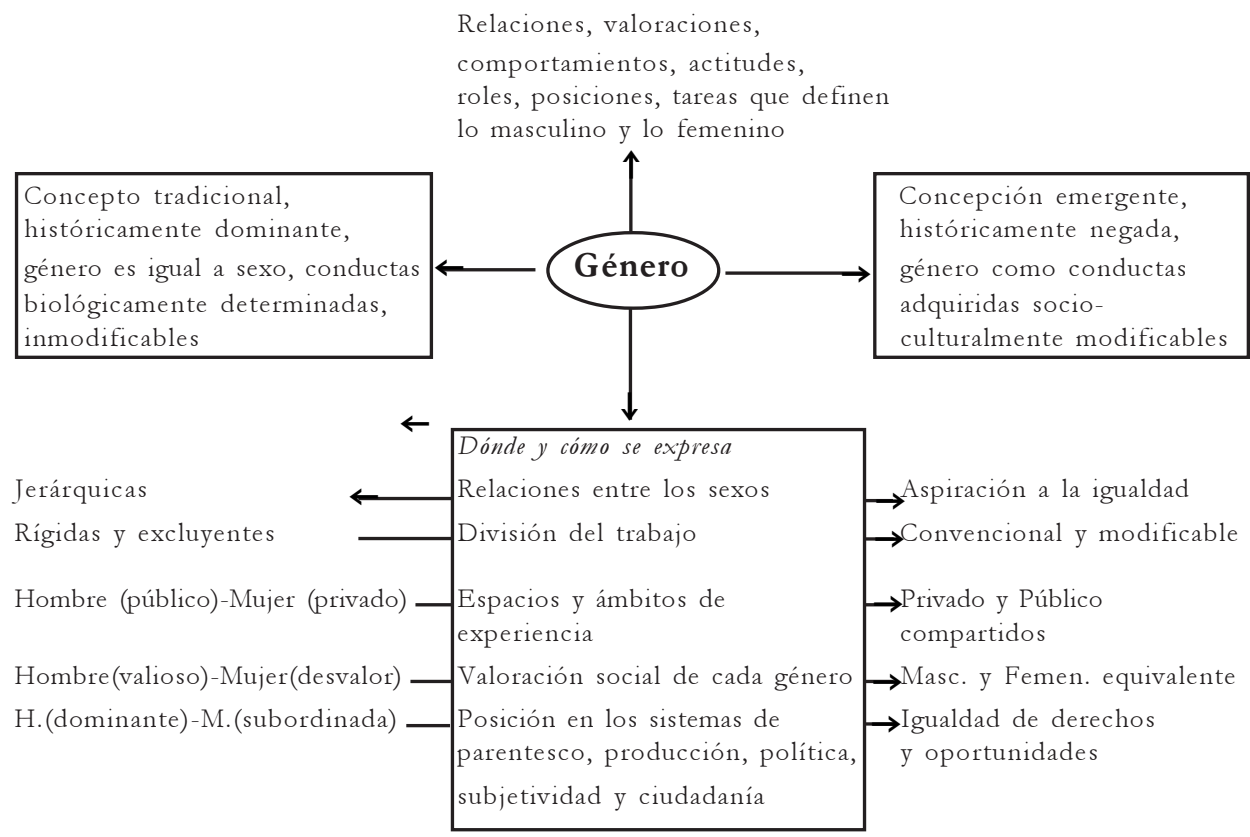

FUENTE: E. García, 1996.

Actualmente la perspectiva de género en el desarrollo "goza de una gran legitimidad internacional". La perspectiva de género trata de tener en cuenta aquellas diferencias que existen entre hombres y mujeres y las relaciones de poder que se establecen entre ellos, en el análisis de la realidad social del país y la comunidad donde van a llevarse a cabo iniciativas de desarrollo. Estos factores determinan las distintas oportunidades que hombres y mujeres tienen para participar en la definición y beneficiarse del proceso de desarrollo (López y Sierra, 2001:17). 
Mirando el desarrollo desde la dimensión de género, según la NORAD (Agencia Noruega para el Desarrollo), es importante comprender la distribución de papeles en la sociedad y cómo estos pueden verse afectados por el proyecto de desarrollo a fin de utilizar los recursos locales de la mejor manera posible y aumentar, por tanto, las posibilidades de éxito del proyecto. Añade que se ha considerado a las mujeres bien como receptoras más o menos pasivas de ayuda, o como un recurso no explotado de la fuerza de trabajo. En muchos casos, el resultado ha sido un uso equivocado de los recursos, mayores cargas de trabajo para las mujeres y un debilitamiento de su posición en la sociedad.

Cuando se habla del papel del género en el desarrollo, generalmente se remite a tres ámbitos: reproductivo, productivo y comunitario. Veamos (Ibid, p.63):

\section{PAPELES DE GÉNERO EN EL DESARROLLO}

\begin{tabular}{|l|l|}
\hline REPRODUCCIÓN & $\begin{array}{l}\text { Tareas necesarias para asegurar la reproducción -es decir, } \\
\text { la renovación- y mantenimiento de la fuerza de trabajo }\end{array}$ \\
\hline PRODUCCIÓN & $\begin{array}{l}\text { Todo el trabajo que produce un excedente, en forma de } \\
\text { productos, pagos o salarios, incluyendo al producción de } \\
\text { alimentos }\end{array}$ \\
\hline GESTIÓN DE LA COMUNIDAD & $\begin{array}{l}\text { Trabajos de mantenimiento de las funciones de la sociedad; } \\
\text { por ejemplo, suministro de agua, servicios sanitarios, } \\
\text { educación. }\end{array}$ \\
\hline
\end{tabular}

El papel de la mujer va -o debe ir- más allá de estas funciones, que si bien son importantes, no hacen realmente a la mujer un actor protagonista del desarrollo, perdiéndose el significado de "género en el desarrollo".

En opinión de Bourqia (1999), el término "género", traducción del término "gender" en inglés, no da cuenta por completo de todo el pensamiento y el análisis que lleva tras de sí, y que consiste en deconstruir, analíticamente, un orden social que se basa sobre el principio de desigualdad de los sexos. La expresión relaciones sociales de sexo y desarrollo tal vez resultaría más apropiada para expresar las relaciones entre los sexos como relaciones producidas y construidas en el seno de una interacción social.

En nuestras previsiones macroeconómicas hablamos de la mujer, pero no de relaciones de género ni de los perjuicios sociales que soportan las mujeres en estas relaciones. Pensar el género y el desarrollo es pensar y concebir un proyecto de sociedad donde se encuentren constantemente esta vigilancia que subraya las relaciones sociales de los sexos. Este proyecto de sociedad debe acompañarse de un proyecto teórico e intelectual que deconstruya estas relaciones sociales de los sexos(Bourgia, 1999:95).

Para Alicia del Olmo, el actual modelo de desarrollo considera como uno de sus retos fundamentales incorporar los conceptos derivados de la teoría de Género como 
instrumentos que faciliten la consecución de la igualdad de derechos entre mujeres y hombres. El enfoque del desarrollo humano sostenible "se centra en la persona, analizando las necesidades y desigualdades derivadas de las relaciones de poder establecidas entre los sexos e intentando crear un contexto más democrático e igualitario" (del Olmo, 2000:130).

Según Itziar Hernández, el debate centrado en las relaciones de poder, del conflicto y las relaciones de género para entender la subordinación de las mujeres, permitió pasar paulatinamente de una concepción de Mujer en el Desarrollo (MED) a Género en el Desarrollo (GED) que está practicando metodologías y herramientas para la planificación (Hernández, 1999:77).

El enfoque de Género en el Desarrollo -añade- además de tratar de satisfacer las necesidades de las mujeres partiendo de un análisis de las relaciones de género en los hogares, comunidad e instituciones, cuestiona el modelo de desarrollo dominante con la alternativa de un desarrollo humano sostenible y equitativo. Veamos (Ibid, p.78):

\section{ASPECTOS PRINCIPALES DE MED Y GED}

\begin{tabular}{|c|c|c|}
\hline & MUJERES EN EL DESARROLLO & GÉNERO EN EL DESARROLLO \\
\hline ENFOQUE & Mujeres como centro del problema. & Desarrollo de mujeres y hombres. \\
\hline TEMA CENTRAI & Mujeres (y niñas). & Relaciones entre mujeres y hombres. \\
\hline \begin{tabular}{l|l} 
PROBLEMA & \\
\end{tabular} & $\begin{array}{l}\text { La exclusión de las mujeres del proceso } \\
\text { de desarrollo (siendo la mitad de los } \\
\text { recursos humanos productivos). }\end{array}$ & $\begin{array}{l}\text { Relaciones desiguales de poder (ricos y } \\
\text { pobres, hombres y mujeres) que frenan } \\
\text { un desarrollo igualitario y la plena } \\
\text { participación de las mujeres. }\end{array}$ \\
\hline OBJETIVO & Desarrollo más eficiente. & $\begin{array}{l}\text { Desarrollo sostenible e igualitario con } \\
\text { toma de decisiones compartidas entre } \\
\text { hombres y mujeres. }\end{array}$ \\
\hline SOLUCIÓN & $\begin{array}{l}\text { Integración de las mujeres en el proces } \\
\text { de desarrollo existente. }\end{array}$ & $\begin{array}{l}\text { "Empoderamiento" de las mujeres y } \\
\text { personas desfavorecidas. Transformación } \\
\text { de relaciones desiguales. }\end{array}$ \\
\hline ESTRATEGIAS & $\begin{array}{l}\text { - Proyectos de mujeres. } \\
\text { - Componente de mujeres. } \\
\text { - Proyectos integrados. } \\
\text { - Aumentar la productividad de las } \\
\text { mujeres. } \\
\text { - Aumentar los ingresos de las mujeres. } \\
\text { - Aumentar las habilidades de las } \\
\text { mujeres para cuidar el hogar. }\end{array}$ & $\begin{array}{l}\text { - Identificar y señalar las necesidades } \\
\text { prácticas de mujeres y hombres para } \\
\text { mejorar sus condiciones de vida } \\
\text { - Al mismo tiempo, identificar y señalar } \\
\text { los intereses estratégicos de las mujeres. }\end{array}$ \\
\hline \begin{tabular}{l|} 
PROBLEMAS \\
CONSECUENCIA
\end{tabular} & $\begin{array}{l}\text { Este enfoque ha aumentado a menudo } \\
\text { la carga de trabajo de las mujeres sin } \\
\text { lograr un mayor poder económico. } \\
\text { Las mujeres no han sido consultadas } \\
\text { sobre el tipo de desarrollo e integración } \\
\text { que buscaban. } \\
\text { Se da una "integración" en el mundo de } \\
\text { de los hombres sin cambios en las rela- } \\
\text { ciones de poder. }\end{array}$ & $\begin{array}{l}\text { Las intervenciones del proyecto se basan } \\
\text { en los roles, responsabilidades y poder } \\
\text { de las mujeres y los hombres en la } \\
\text { sociedad a la que pertenecen y las } \\
\text { necesidades resultantes para cambiar su } \\
\text { situación. Se puede entender GED como } \\
\text { un esfuerzo para mejorar la posición de } \\
\text { las mujeres en relación a los hombres de } \\
\text { manera que beneficie y transforme la } \\
\text { sociedad en su totalidad. }\end{array}$ \\
\hline
\end{tabular}

FUENTE: OXFAM, 1997. 
Es importante esta distinción entre Mujer en el Desarrollo y Género en el Desarrollo que, a menudo, se emplean como sinónimos. Como ya hemos visto difieren en su enfoque, "lo cual tiene implicaciones en las políticas que concluyen en una estrategia política, en la planificación, y en la implementación que es el proceso de acción".

El enfoque de MED tiene ciertos planteamientos, entre ellos (Delso, 1999:158):

- Asimilar el trabajar para transformar las relaciones de género a trabajar con mujeres.

- Considerar que se trabaja con un enfoque de género porque en los proyectos se incluyen acciones específicas para mujeres de distinto tipo: pueden ser formativas, sanitarias, productivas.

- Y en el mejor de los casos, se considera que se cubre ese aspecto porque se trabajan algunos proyectos con contrapartes que son organizaciones de mujeres y/o feministas.

Como expresábamos, este enfoque es superado ya por el enfoque "género y desarrollo" (GED).

La diferencia fundamental entre ambos es que mientras el primero pone el énfasis en la autonomía económica de las mujeres como garantía de igualdad, el segundo trata de transformar las relaciones de desigualdad entre mujeres y hombres, trabajando desde una concepción de sistema, con ambos géneros. Esta perspectiva entiende que las relaciones entre géneros sólo se podrán transformar si se transforma el sistema como un todo, no únicamente alguno de sus elementos(Delso, 1999:158).

En este sentido se consideran elementos clave a transformar la división sexual del trabajo, el acceso y control de los recursos y beneficios y la participación en el poder político o en la toma de decisiones. Para el enfoque GED es fundamental tener en cuenta las relaciones de poder. En palabras de Ajamil:

La concepción de "género y desarrollo" supera la visión de las funciones de hombres y mujeres en la sociedad, para examinar sus roles y especialmente las relaciones entre ellos y su impacto sobre el desarrollo. Se pretende, por tanto, mirar el potencial de las iniciativas de desarrollo para producir cambios en las relaciones sociales y de género, que también faciliten la adquisición de poder por parte de las mujeres (Ajamil, 1999:399).

Es básico anotar que "el enfoque de género y desarrollo concibe los géneros como construcciones culturales, históricas y aprendidas, susceptibles, por tanto, de cambio y de transformación".

El análisis de género insiste en que toda acción de desarrollo (de cualquier naturaleza) tiene un impacto sobre las relaciones de género. La "ceguera de género", o la incapacidad para reconocer que la dimensión de género es un factor clave, "puede convertirse en un obstáculo real al desarrollo sostenible y participativo". 
Con el análisis de género, los planificadores se aseguran de que las acciones de desarrollo basan sus actividades en información real, y no en supuestos o ideas que se asumen por anticipado sobre las vidas de hombres y mujeres. Ayuda a organizar la información de manera tal que permite detectar las necesidades de información adicional y el impacto potencial que la acción propuesta tendrá sobre las mujeres y hombres implicados. Para realizar un análisis de género es necesario que la información esté desagregada por sexos, lo que implica que ésta se agrupa en función de lo que es relevante para las mujeres y sus roles, y para los hombres y sus roles en la sociedad (López y Sierra, 2001:58).

Así, López y Sierra (2001:59) presentan seis elementos básicos en el análisis de género:

a) Perfil de actividades de hombres y mujeres: roles y análisis de distribución del tiempo

b) Acceso y control de recursos y beneficios

c) Factores de influencia en las relaciones de género

d) Identificación de las necesidades e intereses de hombres y mujeres

e) Participación de hombres y mujeres

f) Capacidad de las organizaciones responsables para trabajar con perspectiva de género

Hay que dejar claro qué entendemos por incorporar la perspectiva de género en el trabajo de desarrollo. Desde esta mirada, se contribuye a la "participación equitativa de hombres y mujeres en el diseño, ejecución, toma de decisiones y el acceso, uso y control de los beneficios de las iniciativas de desarrollo". Así, asumir el enfoque de género y desarrollo supone incorporar una nueva manera de mirar la realidad, nuestra realidad.

\section{MUJER Y DESARROLLO}

Hemos visto la diferenciación entre Mujer en el Desarrollo y Género en el Desarrollo. Aquí se resalta la importancia del papel de la mujer en el desarrollo, no como objeto del desarrollo sino como un sujeto activo y participativo en este proceso ${ }^{2}$.

Uno de los objetivos centrales de la cooperación al desarrollo es la lucha contra la pobreza y las desigualdades sociales. En este sentido -en opinión de Santos y Caballero, la mujer, como grupo social y como persona, vive una situación de discriminación, fundamentada en una estructura social de preeminencia masculina en todos sus aspectos sociales, económicos y políticos. Y enfrentar el problema del desarrollo significa luchar contra toda forma de discriminación y potenciar todos aquellos factores económicos y sociales que permitan un crecimiento material y una distribución equitativa de la riqueza.

\footnotetext{
${ }^{2}$ Para una visión más amplia sobre el concepto y teorías del desarrollo ver Arizaldo Carvajal Burbano. Cultura y Desarrollo Local, Documento de Trabajo, Facultad de Humanidades, Universidad del Valle, Cali, 2002. 
En esta perspectiva, "las políticas contra la discriminación de la mujer y su promoción como fuerza social y económica, constituyen un elemento esencial de políticas integrales de desarrollo" (Santos y Caballero, 1994:335).

Las autoras parten de la base de la siguiente tesis:

"La no consideración de la perspectiva de género en los programas y proyectos de desarrollo, supone desconocer una realidad de desigualdad y marginación de un grupo social que constituye más de la mitad de la población, lo que implica una carencia fundamental que cuestiona la viabilidad y eficacia de los objetivos de las políticas de desarrollo" (Ibid, p.336).

Añaden que si se parte de considerar que uno de los objetivos del desarrollo es mejorar el nivel de vida de la población, tendremos que plantearnos que la mejora de la posición de la mujer en la sociedad entra dentro de estos objetivos y para ello se hace necesario ampliar el debate sobre mujer y desarrollo con el fin de producir un nuevo tipo de conocimiento (sobre la construcción de las relaciones de género y la articulación de las mismas) que permitan la elaboración de nuevas estrategias de acción.

Es importante mirar cómo las mujeres perciben, significan, simbolizan el desarrollo; en otras palabras, cómo lo representan. Rivera Garretas hace una interesante observación:

Nombrar el mundo en femenino se refiere a la obra de reconocimiento y de creación de significado de las relaciones sociales hecha a lo largo del tiempo por mujeres. A esta obra de creación de significado, de reconocimiento del sentido del mundo en que vivimos, se le llama hoy día hacer orden simbólico (Rivera Garretas, 1998:11).

Nombrar el mundo -puntualiza- "es una necesidad común de vida que ayuda a que cada una o cada uno de nosotras tenga a raya la insensatez que, acumulada, marca o puede marcar el umbral de la locura. Locura que, cuando es de mujer, ha sido denominada histeria, depresión, miedo indeterminado...".

Así como el género, el desarrollo es también construido socialmente; son "construcciones sociales y culturales". De ahí la importancia de mirar esos sistemas simbólicos o de significado.

En el campo de la Cooperación Internacional, la Comunidad Europea ha diseñado su política "mujer y desarrollo" con dos líneas de acción. En primer lugar, contribuyendo a aportar a la mujer los medios necesarios para mejorar su productividad y sus ingresos, teniendo en cuenta la dimensión de género en el momento de la asignación de los medios de producción, capitales y tecnología, educación, créditos, etc. En segundo lugar, contribuyendo a favorecer el desarrollo de los recursos humanos a través de la educación y los servicios de planificación familiar y sanitarios (Gómez y Sanahuja, 1999:172).

El IV Convenio de Lomé recoge ya estos principios. El 20 de diciembre de 1995, el Consejo aprueba una resolución titulada "Integrar las cuestiones de género en la 
cooperación al desarrollo". En este documento se entiende por género "los papeles diferentes e interrelacionados, responsabilidades y oportunidades del hombre y de la mujer, que son culturalmente concretos y socialmente estructurados y que pueden sufrir modificaciones en el tiempo como resultado de las intervenciones políticas". Los principios que guían a las acciones de cooperación de la Comunidad y de los Estados miembros son los siguientes (Ibid, p.173):

- Los análisis de género en todos sus niveles deben ser centrales en la concepción, diseño y puesta en práctica de todas las intervenciones y políticas de desarrollo, así como en el seguimiento y la evaluación.

- Hombres y mujeres deben participar y beneficiarse del proceso de desarrollo en un plano de igualdad.

- Reducir los desequilibrios de género es una prioridad para la sociedad en su conjunto.

- El análisis de las desigualdades entre hombres y mujeres debe ser un criterio clave a la hora de valorar los fines y los resultados de las intervenciones y políticas de desarrollo.

- La cooperación al desarrollo debe fomentar y apoyar cambios en actitudes, estructuras y mecanismos a los niveles político, legal, comunitario y doméstico en orden a reducir desigualdades de género y en particular:

-Debe promover en todos los niveles una participación completa e igualitaria en el proceso de toma de decisiones y en la distribución del poder político.

-Debe promoverse el empoderamiento económico y el acceso y control igualitario sobre los recursos económicos.

-Debe ser fomentado el acceso igualitario y el control sobre las oportunidades del desarrollo social.

Así pues, un desarrollo liberado del sesgo de género -expresa Bourqia- sería un desarrollo en el que se reunieran el principio de eficiencia -puesto que la presencia de las mujeres en el desarrollo es rentable- y el principio de igualdad de los sexos.

\section{LAS NECESIDADES DE GÉNERO EN EL DESARROLLO}

En el desarrollo con perspectiva de género, se definen dos tipos de necesidades: las prácticas y las estratégicas.

Las necesidades prácticas son aquellas que tienen las mujeres y los hombres dentro de los papeles establecidos y socialmente aceptados en la sociedad. La satisfacción de las necesidades prácticas no contribuirá al cambio -o fortalecimiento- del papel del individuo en la sociedad.

Las necesidades estratégicas, por contraste, describen lo que, de distintas formas, contribuye a fortalecer la situación del individuo en la sociedad (NORAD, 1997:63). 
Veamos (Ibid.) :

NECESIDADES RELATIVAS AL GÉNERO

\begin{tabular}{|l|l|}
\hline NECESIDADES PRÁCTICAS & $\begin{array}{l}\text { Necesidades cubiertas dentro del papel socialmente aceptado } \\
\text { del individuo, tales como: }\end{array}$ \\
\hline - Acceso a artículos de consumo básicos como agua, \\
vivienda, alimentos, etc. \\
- Acceso a servicios sociales básicos \\
- Acceso a un trabajo generador de renta
\end{tabular}

Para Irene López (2000:66), promover el empoderamiento de las mujeres implica, por tanto, enfocar no sólo las necesidades prácticas sino también las estratégicas de forma interrelacionada. La condición y posición de hombres y mujeres en el desarrollo ${ }^{3}$ están intimamente relacionadas y se refuerzan. La posición explica la condición y, al tiempo, las condiciones de vida que contribuyen a mantener las desigualdades en la posición relativa de hombres y mujeres.

Añade que la falta de valoración y la ausencia de representación de intereses, puntos de vista y necesidades de las mujeres repercute directamente en una adecuada identificación de sus problemas en relación al desarrollo.

${ }^{3}$ La condición de las mujeres se refiere a sus condiciones concretas de vida, a sus condiciones materiales y su esfera de experiencia inmediata, expresada en función del trabajo que realiza, el lugar en que vive, sus necesidades y las de las personas que conforman su hogar. La posición se refiere a la desigual ubicación social, política, económica y cultural de las mujeres respecto de los hombres en un contexto determinado. La posición general de las mujeres se manifiesta como subordinada en las relaciones de género (López, 2000:66). 
Son mucho más aceptables los proyectos dirigidos a atender necesidades prácticas de las mujeres, es decir, las que se derivan de sus condiciones concretas de vida, normalmente ligadas a su rol reproductivo y al bienestar de sus familias: alimentación, vivienda, agua, ya que no cuestionan los roles tradicionales de hombres y mujeres, ni su posición relativa. Cuando este tipo de proyectos, dirigidos exclusivamente a las mujeres, no toman en consideración las relaciones entre los géneros, ni los obstáculos institucionalizados que pueden estar perpetuando las desigualdades, se da lo que Marcela Lagarde ha denominado "enfoque aséptico de género", que no enfrenta las causas que están al origen de esa desigualdad estructural de las mujeres (Ibid, pp.67-68).

Dicho "enfoque aséptico de género" ha predominado en muchos programas o proyectos de desarrollo. De ahí que se requiera un cuestionamiento de las relaciones de poder, de la misma estructura social que posibilita estas desigualdades. Y donde las mujeres hablen con su propia voz.

\section{EL EMPODERAMIENTO. LA MUJER COMO ACTOR SOCIAL}

Cuando se habla de empoderamiento, no existe un consenso en torno a su significado, es un concepto utilizado en diferentes sentidos, "y en muchas ocasiones son aplicados de forma retórica, vaciándoles del contenido político que les da sentido".

La estrategia del empoderamiento y la de mainstreaming (integración en la

"corriente principal"), son respuestas a los dos principales problemas que se han identificado en el pensamiento feminista durante la aplicación de las políticas de "Mujeres en el Desarrollo" (MED) en las últimas décadas. El primero es que si bien estas políticas han conseguido en cierta medida mejorar las condiciones materiales de vida de las mujeres, han sido mucho menos efectivas en lo que se refiere a la mejora de su poder económico y social respecto de los hombres en el contexto del desarrollo. Y el segundo es la persistente marginalización política de las opiniones y puntos de vista de las mujeres en el proceso de desarrollo, especialmente a nivel de la planificación del desarrollo en instituciones tales como las burocracias estatales y los organismos de cooperación para el desarrollo, desde los multilaterales hasta las ONGD (López Méndez, 2000:59-60).

Estos dos conceptos estratégicos - empoderamiento y mainstreaming- se han ido consolidando para la búsqueda de una mayor igualdad entre los hombres y las mujeres. 
GRÁFICA No. 2

LAS DOS ESTRATEGIAS PARA LA IGUALDAD

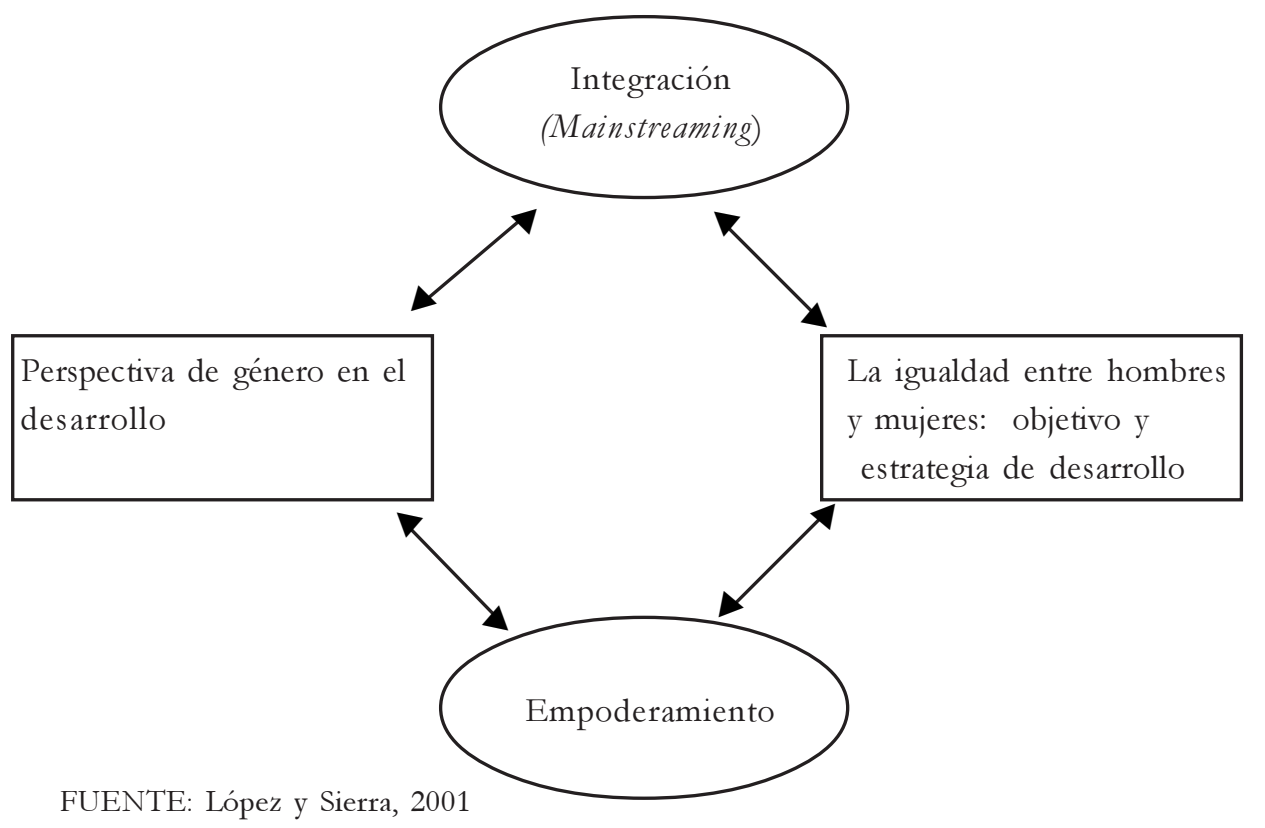

Ya el llamado empoderamiento fue adoptado como estrategia clave de desarrollo en la Declaración de Pekín (1995):

El empoderamiento de las mujeres y su plena participación en condiciones de igualdad en todas las esferas de la sociedad, incluyendo la participación en los procesos de toma de decisiones y el acceso al poder, son fundamentales para el logro de la igualdad, el desarrollo y la paz (epígrafe 13).

Pero, se pregunta López, ¿Qué es el empoderamiento y cómo determinar la virtualidad de una acción de desarrollo para el mismo?

Lo primero que hay que tener claro es que no se puede "empoderar" a las personas. Se trata más bien de programas e intervenciones que contribuyan a crear las condiciones necesarias para promover que las mujeres sean agentes de su desarrollo y empoderamiento. Las agencias de desarrollo y las ONG, en particular, pueden apoyar procesos que aumenten la autoestima de las mujeres, que las hagan más autónomas y les ayuden a establecer sus propios objetivos(López Méndez, 2000:60).

También, para la autora, el empoderamiento señala el reconocimiento por parte de aquellos que trabajan en el nivel local, más allá de la retórica del desarrollo participativo, de que el poder de definir prioridades debe encontrarse donde siempre estuvo, en las 
manos de los excluidos. Así, el concepto de empoderamiento está claramente vinculado a la noción de poder y su reverso: la falta o ausencia de poder.

Además, es fundamental poner de manifiesto los términos de la participación de los distintos grupos involucrados. Puede haber muy distintos grados y calidades en la participación de hombres y de mujeres. Sólo algunos espacios e iniciativas permiten realmente la implicación de las mujeres y su reconocimiento como agentes de su propio desarrollo. A ello hay que sumar, en coherencia con la perspectiva de género en el desarrollo, que el proceso de empoderamiento también involucra a los hombres (Ibid, pp.61-63).

Un aspecto central del empoderamiento es la participación de las mujeres en la determinación de necesidades, así como en los distintos espacios de poder que se generan en torno a las políticas, programas y proyectos de desarrollo. "Son las propias mujeres las que han de determinar cuáles son sus necesidades y esto sólo es posible mediante la generación de un espacio a través del cual puedan articularlas, lo que no siempre se identifica y apoya". Las mujeres, al igual que cualquier grupo de menor influencia en la comunidad, tienen puntos de vista, necesidades e intereses propios, aunque tengan obstáculos de muy distinta naturaleza para expresarlos (Ibid, p.68).

Permitir a las mujeres hablar en nombre propio sobre sus propias prioridades y vidas, no sólo ayudará a sacarlas de su condición de eternas víctimas enmudecidas, sino que además tiene implicaciones muy prácticas para el empoderamiento (Ibid, p.69).

No hay que olvidar que los hombres deben asociarse también a ese proceso de cambio.

El empoderamiento no debe concebirse como un juego de suma cero donde las ganancias de las mujeres automáticamente implican pérdidas para los hombres.

Hay ganancias del empoderamiento de las mujeres para la sociedad y para los hombres que hay que identificar y difundir (Ibid, p.70).

Y es importante recordar que las mujeres no son un grupo homogéneo. Si bien las mujeres pueden compartir intereses comunes basados en su pertenencia al género femenino, la clase, la raza, la edad y la religión también determinan los intereses de las personas. Las vidas, experiencias, necesidades e intereses de las mujeres "son complejos y acabar con la subordinación de las mujeres es un proyecto político multifacético y de largo alcance que tiene en su centro no sólo a las mujeres sino también a los hombres" (Ibid, p.78).

Algunos autores analizan el empoderamiento con doble significado: por una lado "dar poder" /"conceder a alguien el ejercicio del poder" y, por otro, "ganar o tomar el poder", considerándose esta última acepción como obsoleta. En el ámbito institucional prevalece la idea de dar poder. 
En general, se parte de la idea que es posible construir el empoderamiento a través de una planificación específica dirigida a mujeres. Esta línea de acción corresponde a lo que Angela Cheater ha descrito como la transferencia de empoderamiento a través de las agencias, un discurso que es dominante en el pensamiento sobre desarrollo (Madrigal, 2000:89).

Es una transferencia desde arriba -desde los espacios de poder- hacia abajo -hacia la gente desempoderada. Esta transferencia de empoderamiento se considera finalizada una vez que las mujeres estén empoderadas, es decir, cuando estén en la posición de toma de decisiones y, más concretamente, cuando estén representadas en el poder institucional.

Por otro lado -agregan los autores-, fuera del discurso de las instituciones internacionales aparece otra visión de cómo lograr el empoderamiento.

Esta visión, que gira alrededor del empoderamiento entendido como self-empowerment, o autoempoderamiento, es opuesta al enfoque anterior. Desde esta óptica el empoderamiento es un proceso político, un proceso nunca acabado donde caben nociones como autoestima, desarrollo personal, dignidad, autonomía y concienciación. Se trata de un proceso de crecimiento individual como colectivo a través de la participación en colectivos o movimientos sociales, donde las redes juegan un papel fundamental (Ibid.).

Una visión que sí considera que el proceso de empoderamiento puede crear mucha resistencia por parte de aquellas personas o grupos sociales que corren el riesgo de desempoderarse.

En desarrollo, el empoderamiento -como fuerza transformadora- no debe dejar de lado su indudable cariz político y de cambio.

\section{CONCLUSIONES}

Comparto la opinión de López y Alcalde de que "trabajar los problemas del desarrollo desde una perspectiva de género implica una toma de postura ante la realidad no exenta de riesgos y obstáculos".

Implica, sobre todo, un posicionamiento ético y político. Ético porque se fundamenta en valores tan fuertes como la equidad y la justicia y político porque rechaza el asepticismo y no se conforma con la realidad existente: vive y cobra sentido a través de la intervención contra la desigualdad, la deconstrucción de las estructuras que la mantienen y el cambio hacia una sociedad que reconozca la paridad en la diferencia (López y Alcalde, 1999:7).

Añaden que las mujeres han estado excluidas de la construcción de la cultura, el pensamiento, la política. Y que "su contribución al desarrollo de sus países ha sido in visibilizada y la conformación del modelo de desarrollo predominante ha sido ajena a 
sus experiencias y puntos de vista".

Hay que anotar que se ha avanzado en un amplio reconocimiento del rol de la mujer en los procesos de desarrollo, "así como en la visibilidad de las mujeres en las estadísticas e investigaciones".

Decíamos que diversas instituciones internacionales -y locales- están jugando un papel importante con el fin de eliminar las desigualdades existentes entre los hombres y las mujeres en los espacios de poder, con el objetivo de promover la igualdad de género. En el marco de sus políticas de género la palabra empoderamiento aparece como una necesidad en las líneas de acción.

Este proceso exige la participación plena de mujeres y de hombres, de todos los grupos diversos de beneficiarios de los proyectos. Este requisito refuerza aún más los argumentos en favor de los principios de democracia y desarrollo participativo que toda acción de desarrollo debe fomentar. Según Marcela Lagarde, "no hay desarrollo humano sin las mujeres y que la democracia implica, en primer término, la democracia genérica".

Como bien lo señala Itziar Hernández "a pesar de las dificultades, la incorporación de las mujeres en el desarrollo es un proceso imparable como lo es el aumento de grupos de mujeres en todos los países del mundo que están trabajando en la construcción de una alternativa al modelo de desarrollo imperante".

\section{BIBLIOGRAFÍA}

AJAMIL, MENCHU (1999). "Enfoques y estrategias sobre género y desarrollo", en Paloma de Villota (Editora). Globalización y género, Editorial Síntesis, Madrid. BOURQIA, RAHMA (1999). "Género y desarrollo", en Dirección General de la Mujer. Mujeres y desarrollo. II Encuentros Euromediteráneos para el Desarrollo, Comunidad de Madrid.

CARVAjal BURBano, ARIZALdo (2002). Cultura y Desarrollo Local, Documento de Trabajo, Facultad de Humanidades, Universidad del Valle, Cali.

CASTELlanOS, GABRIELA (2002). "Introducción: Breve meditación sobre cuerpo y vestido, género y sexo”, en Gabriela Castellanos y Simone Accorsi (Compiladoras). Género y sexualidad en Colombia y en Brasil, Universidad del Valle, Cali.

CRUZ, CARMEN DE LA (1998). Guía metodológica para integrar la perspectiva de género en proyectos y programas de desarrollo, Instituto Vasco de la Mujer, Vitoria.

DELSO ATALAYA, ANA (1999). "La incorporación del enfoque de género en el trabajo de las organizaciones no gubernamentales de desarrollo”, en Irene López y Ana Rosa Alcalde(coords). Relaciones de género y desarrollo. Hacia la equidad de la cooperación, Los Libros de la CatarataIUDC/UCM, Madrid.

DIRECCIÓN GENERAL DE LA MUJER (1999). Mujeres y desarrollo. II Encuentros Euromediteráneos para el Desarrollo, Comunidad de Madrid.

Escobar, ARTURo (1998). La invención del Tercer Mundo. Construcción y deconstrucción del desarrollo, Editorial Norma, Santafé de Bogotá.

GIMENO, JUAN CARLOS y PILAR MONREAL (eds) (1999). La controversia del desarrollo. Críticas desde la antropología, Los libros de la Catarata-IUDC/UCM, Madrid.

GÓMEZ GALÁN, MANUEL y JOSÉ ANTONIO SANAHUjA (1999). El sistema internacional de cooperación al desarrollo. Una aproximación a sus actores e instrumentos, CIDEAL, Madrid. 
Goulet, Denis (1999). Etica del desarrollo. Guía Teórica y Práctica, IEPALA Editorial, Madrid. HERNÁNDEZ ZUBIZARRETA, ITZIAR (1999). "Desigualdad de género en desarrollo", en Paloma de Villota (Editora). Globalización y género, Editorial Síntesis, Madrid.

LAGARDE, MARCEla (1997). Género y feminismo. Desarrollo humano y democracia, Horas y horas, Madrid.

LÓPEZ MÉNDEZ, IRENE (2000) . "Empoderamiento y mainstreaming: estrategias para la igualdad entre los géneros", en Revista Española de Desarrollo y Cooperación No. 6. IUDC/UCM, Madrid. Primavera/Verano 2000.

LÓPEZ, IRENE y ANA ROSA ALCALDE(coords) (1999). Relaciones de género y desarrollo. Hacia la equidad de la cooperación, Los Libros de la Catarata-IUDC/UCM, Madrid.

LÓPEZ MÉNDEZ, IRENE y SIERRA LEGUINA, BEATRIZ (2001). Integrando el análisis de género en el desarrollo. Manual para técnicos de cooperación, IUDC/UCM-AECI, Madrid.

MADRIGAL, PALOMA ET AL (2000). "El empoderamiento en la cooperación al desarrollo: dudas y reflexiones”, en Revista Española de Desarrollo y Cooperación No. 6. IUDC/UCM, Madrid. Primavera/Verano 2000.

MINISTERIO DE ASUNTOS EXTERIORES-SECIPI (1998). Metodología de evaluación de la Cooperación Española, Madrid.

moser, Carolina (1995). Planificación de género y desarrollo. Teoría, Práctica y Capacitación, Flora Tristán, Perú.

NORAD (Agencia Noruega para el Desarrollo) (1997). Evaluación de proyectos de ayuda al desarrollo. Manual para evaluadores y gestores, IUDC-UCM/CEDEAL, Madrid.

OLMO GARRUDO, ALICIA DEL (2000). "Una aproximación a los recursos bibliográficos básicos sobre género y desarrollo”, en Revista Española de Desarrollo y Cooperación No. 6. IUDC/UCM, Madrid. Primavera/Verano 2000

OSTERGAARD, LISA (coord) (1991). Género y desarrollo. Guía práctica, MAS-INSTITUTO DE LA MUJER, Madrid.

PNUD (Programa de las Naciones Unidas para el Desarrollo). Género y desarrollo bumano: el reto de los próximos siglos (Internet).

Rivera garretas, maría milagros (1998). Nombrar el mundo en femenino. Pensamiento de las mujeres y teoría feminista, Icaria Editorial, Barcelona.

SANTOS, ANTONIA y CLARA CABALLERO (1994). "Mujer y desarrollo: Cooperación internacional, perspectiva de género y políticas de desarrollo", en Guzmán Alonso et al. Nuevas tendencias en la cooperación internacional, CIDEAL, Madrid.

SIERRA LEGUINA, BEATRIZ (2000). "Criterios para la evaluación con perspectiva de género", en Revista Española de Desarrollo y Cooperación No. 6. IUDC/UCM, Madrid. Primavera/ Verano 2000

villota, Paloma dE (Editora) (1999). Globalización y género, Editorial Síntesis, Madrid. 